\title{
Einfluss der kolloidalen Lösung auf die Rückresorption des Kochsalzes in der Niere.
}

(Studien über Flüssigkeitsaustausch. XXXXI.)

\author{
Von \\ Koichi Aida. \\ (會田宏一) \\ (Aus der Medizinischen Klinik von Prof. Dr. T. Kato, \\ Tohoku Reichsuniversität zu Sendai.)
}

In meiner vorherigen Mitteilung') ist an isolierten überlebenden Krötennieren unter Ausschaltung extrarenaler Faktoren nachgewiesen worden, dass kolloidale Lösung eine bedeutende Verminderung der Harnbildung bewirkt. In vorliegender Arbeit ist die Durchspülung der Krötenniere mit der Ringerlösung mit Rinderserumzusatz ausgeführt worden, um darüber zu untersuchen, welche Einflüsse eine derartige kolloidale Lösung auf die intrarenale Harnabsonderung, vorzüglich auf den Kochsalzgehalt im Harn ausübt.

Was die Verschiebung der im Harn auszuscheidenden NaCl-Konzentration sowie das Verhalten der Harnmenge anbelangt, liegen diesbezügliche literarische Angaben von verschiedenen Autoren vor. Es haben nämlich Thompson ${ }^{2}$ am Hund, Frey ${ }^{3)}$ am Kaninchen durch intravenöse Injektion von hypertonischer Kochsalzlösung Diuresé sowie eine Abnahme des NaCl-Gehaltes im Harn wahrgenommen, Ma g$n u s^{4)}, A$ be u. Sakata $a^{5}$ hingegen an Kaninchen unter gleichen Bedingungen nebst der Diurese eher eine Zunahme der NaCl-Konzentration nachgewiesen. Yoshida ${ }^{6)}$, Deuts ch ${ }^{7)}$ Schurmyer ${ }^{8)}$ haben bei

I) A i d a, Tohoku Journ. Exper. Med., 1943, 45, 250.

2) Th o mpso n, Journ. Physiol., 1899, 25, 487.

3) Fre y, Pfügers Arch., 1907, 120, 90.

4) Magnu s, Arch. f. Exp. Pathol. u. Pharm., 1900, 44, 68.

5) A be u. Sakata, Ibid., 1925, 105, 93.

6) Yoshid a, Pflügers Arch., 1924, 206, 274.

7) Deutsch, Ibid., 1925, 208, 177.

8) Sehurmyer, Ibid., 1925, 210, 759. 
Durchspülung isolierter überlebender Froschniere Oligurie sowie den allmählichen Anstieg des Kochsalzgehaltes im Harn konstatiert. Obengenannte Autoren behaupten ubereinstimmend, dass die Niere die Verdünnungsarbeit leiste. Bainbridge, Collins u. Menzies ${ }^{9)}$ fanden, dass der Harn dann mit der Spülflüssigkeit isotonisch wurde, wenn die Kanälchenwandung der Froschniere durch Vergiftung mit Sublimat ausgeschaltet wurde. Yoshid $a^{6}$ gibt an, dass das in Normal-Ringer enthaltene $\mathrm{NaCl}$ im sog. Harn um $30 \%$ verdünnt erscheine; er erklärt dies durch eine auf beeinträchtigter Nierenepithelfunktion beruhende Herabsetzung der Rückresorption. Takeda $\mathrm{a}^{10}$, der isolierte überlebende Krötenniere mit hypertonischer und auch hypotonischer Ringerlösung durchströmte, fand in den beiden Fällen das Auftreten der Oligurie; in der Folge sah Iz umida ${ }^{11}$, der auch einschlägige Versuche mit 1,2\% iger $\mathrm{NaCl}$-Ringerlösung angestellt hat, zusammen mit der Oligurie den Kochsalzgehalt im Harn allmählich ansteigen; dies Phänomen hat er auf eine Herabsetzung der Nierenepithelfunktion, die Rückresorption zu regulieren, zurückgeführt und somit einen Beleg zur Yamagu ch is ${ }^{12)}$ Vorstellung geliefert.

In vorausgegangener Mitteilung.1) wurde darauf hingewiesen, dass Kolloide auf die intrarenale Harnbildung einen bestimmten Einfluss ausüben; in vorliegender Mitteilung habe ich auch nach gleichem Verfahren wie in vorheriger Mitteilung ${ }^{1)}$ die Frage in Angriff genommen; was für einen Einfluss das Kolloid auf die Rückresorption und die Ausscheidung des $\mathrm{NaCl}$ ausüben würde.

In vorliegenden Versuchen wurden, indem man isolierte überlebende Krötennieren in gesunden sowie pathologischen Zuständen einerseits von dem glomerulären System aus mit der Normal-Ringerlösung anderseits von der renoportalen Seite aus mit einer Ringerlösung, die, wie später erwähnt werden soll, mit dem Rinderserum versetzt wurde, durchspuilte, das Verhalten der Harnmenge und auch die Verschiebung der Kochsalzkonzentration im Harn analysiert, um dadurch zum Problem der Nierenfunktion beitragen zu können.

Versuchsmethode: Versuchsmethode war dieselbe, wie sie in vorheriger Mitteilung ${ }^{1)}$ angewendet wurde; als Versuchstiere wurden solche Kröten, welche sich im Zustand des Sommerlebens, befanden, benutzt. Zwecks Erzeugung der Nierenschädigungen wurden Uranylnitrat sowie Kantharidin angewandt. Die Herstellung der iso-

9) Bainbridge, Colins n. Menzies, Journ. Physiol., 1914, 48, 233.

10) Takeda, Tohoku Journ. Exp. Med., 1935, 27, 281.

11) I z u mid a, Ibid., 1939, 36, 82.

12) Y a maguchi, Ibid., 1930, 16, 361. 
lierten überlebenden Nierenpräparate und das Durchspülungsverfahren derselben sind in der IX. Mitteilung ${ }^{13)}$ einschlägiger Studien ausführlich beschrieben worden.

Als kolloidale Lösung wurde die Rinderseum-Ringerlösung benutzt, die in der Weise hergestellt wurde, dass das Rinderseum, welches zuerst von Fibrin befreit und dessen Eiweissgehalt danach refraktometrisch bestimmt wurde, unmittelbar vor Versuchsanstellung' mit der Ringerlösung derart vermischt wurde, dass die verfertigte Rinderserum-Ringerlösung ungefähr $3 \%$ Eiweiss enthielt; dieselbe wurde als Spülflüssigkeit* verwendet.

Der Uretertropfen, welcher als Blasenharn aufzufassen ist, wurde alle 10 min in einem kleinen Kölbchen gesammelt; die quantitative $\mathrm{Be}$, stimmung des Kochsalzes wurde nach der Methode von Rusznyak ${ }^{14)}$ durchgeführt.

\section{Versuchsergebnisse.}

An je ein und demselben Versuch wurden dreimalige Parallelbestimmungen vorgenommen.

I. Vorbereitender Versuch: Renoportale Durch: spülung mit Normal-Ringerlösung.

1. Versuch an der gesunden Niere.

Fig. 1. Vorbereitender Versuch (Gesunde Niere). Versuch 1. Durchspülung mit Normal-Ringer von der Nierenarterie u.-pfortader aus.

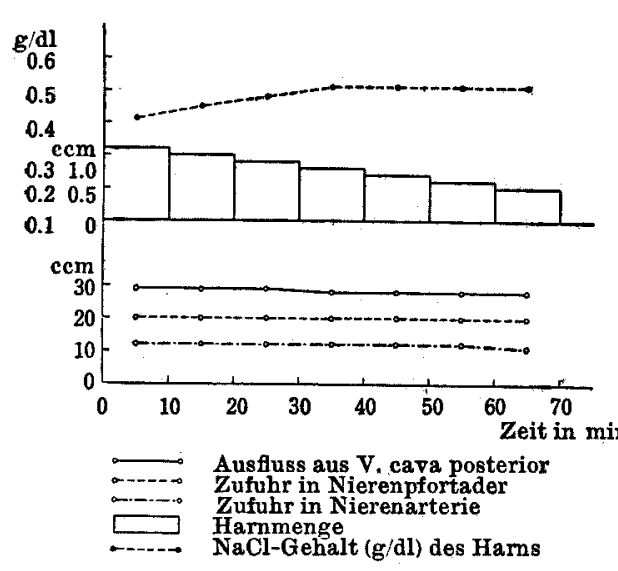

13) S a ito u. Tad a, Tohoku Journ. Exp. Med., 1930, 15, 91.

* Die anf obige Weise hergestellte Spülflüssigkeit wird im folgenden kurz als Rinderserum-Ringerlösung oder schlechthin als Serum-Ringer bezeichnet.

14) Rusznyak, Biochem. Zeitschr., 1921, 114, 23.
Einer von vorliegenden Versuchen ist beispielweise mit Fig. 1 illustriert.

Die Harnmenge nahm mit dem Zeitablauf immer stärker ab, während die NaCl-Konzentration sich allmählich steigerte, um ca. $40 \mathrm{~min}$ danach konstante Werte aufweisen. Während die $\mathrm{NaCl}-\mathrm{Konzentration} \mathrm{der}$ Ringerlösung, der Spülflüssigkeit, $0,6 \%$ betragen hatte, bewegte sich die $\mathrm{NaCl}$ Konzentration des sog. Harnes aber zwischen 0,4 
und $0,5 \%$. Es zeigte sich nämlich, dass die NaCl-Konzentration des sog. Harnes mehr verdünnt als die der Ringerlösung ausgeschieden wurde. Demnach scheint auch ein Krystalloid, wie es auch das Kochsalz ist, beim Abfiltriertwerden durch die Glomeruli eine gewisse Beschränkung zu erfahren. Die Zufuhrmenge von dem Glomerulus aus zeigte die Tendenz zu mehr oder minder Abnahme, doch keine nennenswerte Veränderung.

\section{Versuch an der Uranniere.}

Im ganzen genommen liegen die Verhältnisse ähnlich, wie bei der gesunden Niere; die $\mathrm{NaCl}$-Konzentration war etwas grösser als bei der gesunden Niere, sie betrug nämlich $0,47-0,53 \%$ und zudem steigerte sich mit dem Zeitablauf; die später erfolgte Neigung zur Stetigkeit trat sehr verzögert ein. Die Harnmenge erwies sich schon von Anbeginn an als spärlich, der Grad der Oligurie war erheblich stärker, als bei der gresunden Niere. Die Zufuhrmenge verhielt sich in beinahe analoger Weise, wie bei der gesunden Niere. (Fig. 2).

\section{Versuch an der Kan- tharidinniere.}

$\because \quad$ Im grossen und ganzen wurde hier ein ähnliches Bild angetroffen, wie bei der gesunden Niere. Die NaClKonzentration war aber am höchsten, betrug nämlich $0,48-0,55 \%$, und sie wuchs
Fig. 2. Vorbereitender Versuch (Uranniere). Versuch 1. Durchspülung mit NormalRinger von der Nierenarterie u.-pfortader aus.

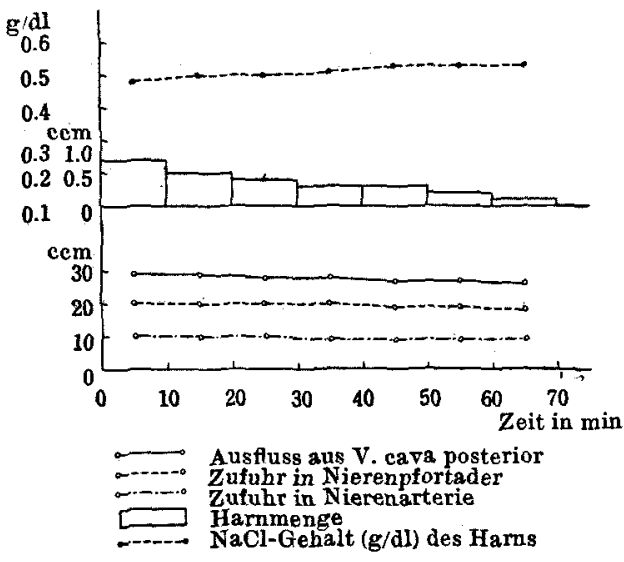

Fig. 3. Vorbereitender Versuch (Kantharidinniere). Versuch 1. Durchspülung mit Normal-Ringer von der Nierenarterie a.-pfortader ans.

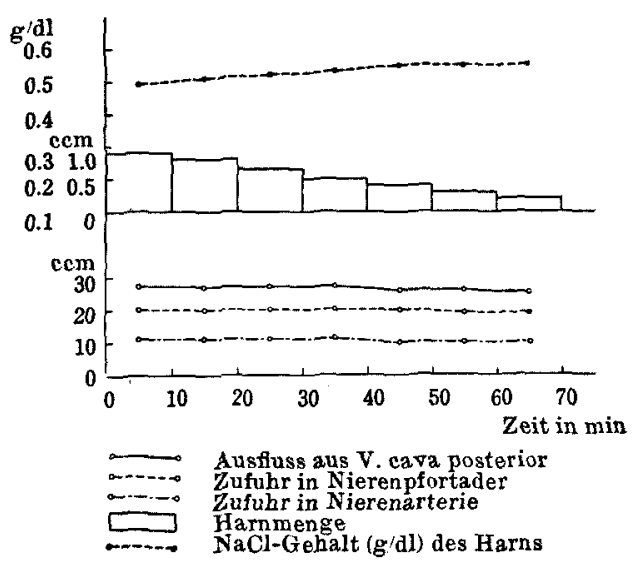


mit dem Zeitablauf immer mehr zu; der Grad dieser Konzentrationssteigerung übertraf denselben sowohl bei der gesunden wie auch bei der Uranniere. Die später erfolgte Neigung zum Konstantwerden trat beträchtlich verzögert ein. Die Harnmenge war spärlicher als bei der gesunden Niere und die mit dem Zeitablauf erfolgte Abnahme der Harnmenge war höhergradig. Die Zufuhrmenge verhielt sich in nahezu. ähnlicher Weise wie bei der gesunden Niere. (Fig. 3).

\section{Einfluss der renoportalen Durchspülung mit Rinderserum-Ringerlösung auf die Kochsalzrückresorption.}

1. Versuch an der gesunden Niere.

Zwecks Vereinfachung der Erörterung wird im folgenden der Zeittraum, wo die Durchspülung mit Normal-Ringer allein von dem glomerulären und renoportalen Kreislauf aus ausgeführt wurde, als das I. Stadium, derjenige Zeitraum, wo die Durchsptulung von dem glomerulären aus mit Normal-Ringer, von dem renoportalen Kreislauf aus aber mit Rinderserum-Ringer ausgeführt wurde, als das II. Stadium bezeichnet.

In vorliegender Versuchsreihe wurde in allen 5 Versuchen im II. Stadium neben erheblicher Abnahme der Harnmenge eine bedeutende Steigerung der NaCl-Konzentration im Harn angetroffen, welche

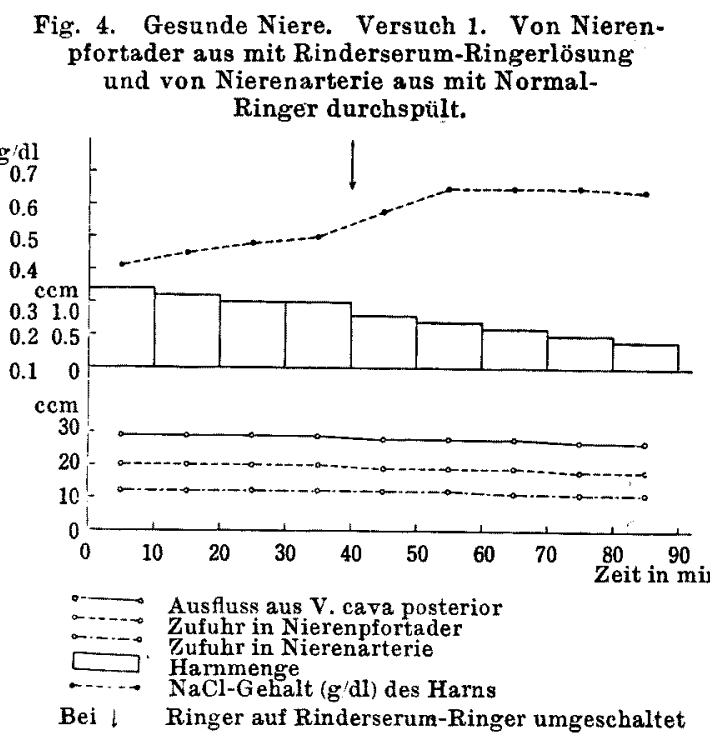
$0,63-0,67 \%$ erreichte. Während die Harnmenge mit dem Zeitablauf allmählich, doch immer stärker abnahm, erwies sich die NaCl-Konzentration schliesslich, als stationär. Die Zufuhrmenge von der Nierenarterie aus hatte mit dem Zeitablauf die Tendenz zu mehr oder weniger Abnahme, jedoch wurde bemerkbare Gefässwirkung vermisst. An der Nie- 
renpfortader war im II. Stadium eine ganz geringfügige Gefässwirkung erkennbar. (Fig.4).

\section{Versuch an der Uranniere. '}

In vorliegender Versuchsreihe wurden 3 Versuche angestellt. Ein Versuch davon ist beispielhalber mit Fig. 5 illustrirt. Die Veränderung im I. Stadium war nicht verschieden von derselben im vorbereitenden Versuch, aber sofort nach dem Übergang in das II. Stadium trat erhebliche Oligurie auf, wobei die $\mathrm{NaCl}-\mathrm{Kon}$ zentration bis auf $0,67-0,69 \%$ anstieg; am Ende des II. Stadiums tendierte die $\mathrm{NaCl}-$ Konzentration zu allmählicher Herabsetzung. Die Harnmenge erwies sich schon von dem Versuchsbeginn an als sehr spärlich, die Intensität der Oligurie übertraf weitgehend die bei der gesunden sowie bei der Kantharidinniere. Die Zufuhrmenge verhielt sich in beinahe gleicher Weise, wie bei der gesunden Niere.

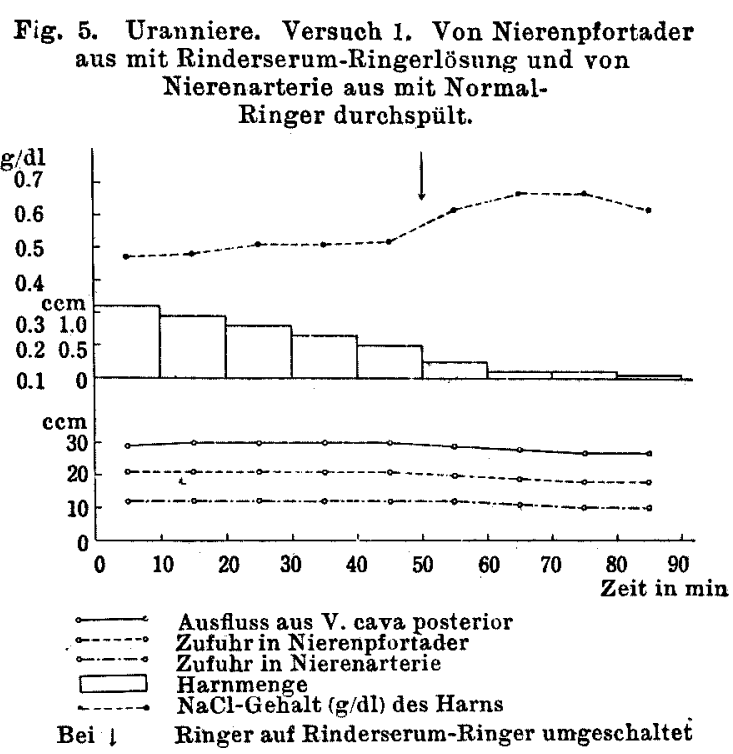

Fig. 6. Kantharidinniere. Versuch 2. Vor Nierenpfortader aus mit Rinderserum-Ringerlösung und von Nierenarterie aus mit Normal. Ringer durchspült.

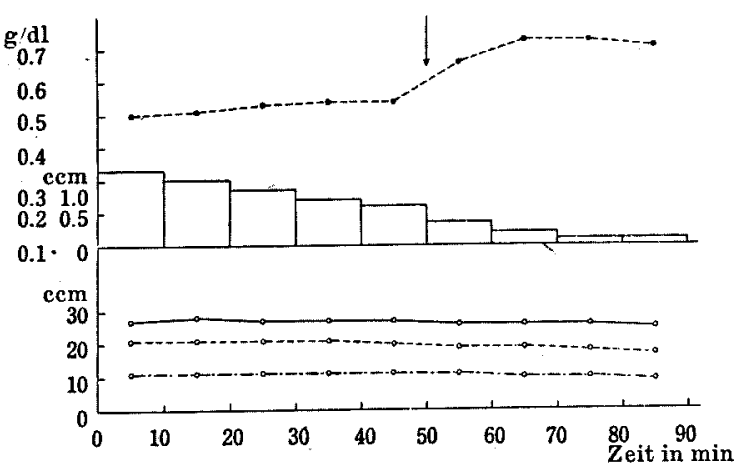

Ausfluss aus V. cava posterior Zufuhr in Nierenpfortader Zufuhr in Nierenarterie Harnmenge NaCl-Gehalt (g/dl) des Harns

Bei $\downarrow$ Ringer auf Rinderserum-Ringer umgeschaltet 


\section{Versuch an der Kantharidinniere.}

Hierbei wurden 3 Versuche durchgeführt (Fig: 6). Im II. Stadium kam ausnahmslos nebst der Oligurie die Steigerung der NaCl-Konzentration zum Vorschein, die 0,64\%-0,75\% erreichte. Die Oligurie nahm mit dem Zeitablauf immer stärker an Intensität zu, die NaCl-Konzentration hielt aber nicht immer mit dieser Intensitätzunahme gleichen Schritt, sondern hatte mitunter eher die Neigung zu allmählicher Stetigkeit. Auch hier verhielt sich die Zufuhrmenge in analoger Weise wie bei der gesunden Niere.

Wenn man unter Übersicht über alle oben angeführte Versuchsergebnisse im besonderen Daten, welche sich bei vorbereitender Durchspülung mit Normal-Ringer allein ergaben, ins Auge fasst, so erkennt man, dass bei isolierten überlebenden Nieren überhaupt im Verein mit der Oligurie eine bestimmte Steigerung der NaCl-Konzentration zutage tritt. Diese Konzentrationssteigerung ist bei der gesunden Niere mindestgradig, bei der Kantharidinniere höchstgradig und bei der Uranniere mittelgradig.

Bei renoportaler Durchspülung mit Rinderserum-Ringer treten in jedem Fall stets erhebliche Oligurie sowie die Steigerung der NaClKonzentration im Harn auf, die über dieselbe in der Spülflüssigkeit hinausgeht. Die Intensität der Oligurie ist bei pathológischen Nieren, namentlich bei der Uranniere, deren Tubuli bekanntlich am stärkstengeschädigt sind,-am ausgeprägsten, daran schlissen sich die Kantharidinniere und gesunde Niere der Reihe nach an. Dër Grad der Hyperchlorurie ist bei der gesunden Niere an niedrigsten, bei der Uranniere und Kantharidinniere der Reihe nach immer höher. Bei in vorheriger Mitteilung ausgeführter Durchspülung mit Gummi-Ringerlösung ist eine geringfügige Gefässwirkung angetroffen worden; auch bei Durchspülung mit Rinderserum-Ringer ist eine äusserst unbedeutende Gefässwirkung an der $\mathrm{V}$. renoportalis nachweisbar.

Oben geschilderte Versuchsergebnisse können nach Yamagu$\mathrm{ch} \mathrm{is}^{12)}$ Ansicht, derzufolge den Tubuluszellen die Funktion, die Rückresorption des Glemerulusharnes zu regulieren bzw. einzuschränken, zukomme, ohne weiteres erklärt werden.

Während im Kontrollversuch irgendwelche Gefässwirkung nicht angetroffen wurde, kommen hier mit dem Zeitablauf eine Abnahme der Harnmenge und eine Steigerung der NaCl-Konzentration zustande; es ist dies sicherlich darauf zurückzuführen, dass bei isolierten 
überlebenden Präparaten auch selbst bei Durchspülung mit NormalRinger die Zellaktivität der Niere selbst durch den mit dem Zeitablauf sich immer stärker geltend machenden Sauerstoffmangel beeinträchtigt worden ist. Weil nämlich im Glomerulus die Schwelle für die Filtration des $\mathrm{NaCl}$ mit dem Zeitverlauf allmählich herabgesetzt ist, dürfte das Kochsalz vermehrt ausgeschieden werden, und zugleich aber auch in den Tubulusepithelien ist ihre beschränkende bzw. regulierende Kraft auf die Rückresorption nach und nach beeinträchtigt, und da Wasser viel leichter rückresorbierbar als das Kochsalz ist, tritt die Verminderung der Harnmenge mit dem Zeitablauf dominierend in Erscheinung. Nach Ya maguch is ${ }^{15)}$ Ansicht führt im intermediären Flüssigkeitsaustausch die Störung der Endothelfunktion zu pathologisch gesteigerter Durchlässigkeit der Kapillaren und da auch in der Niere, die dem Kapillarsystem zugehört, dann, wenn die Glomerulusschlingen funktionell geschädigt sind, eine abnorme Steigerung der Durchlässigkeit für die Lösungen zustande kommt, sollte es nicht zur Abnahme, sondern vielmehr zur Zunahme der Harnbildung kommen. Auch bei bestehender Schädigung der Nierenepithelien müssen diese selbst durch Herabsetzung ihrer Funktion mehr permeabel geworden sein, mit anderen Worten, es läuft letzten Endes darauf hinaus, dass die im Tubuluslumen sich vollziehende Rückresorption eine Steigerung erfährt. Die Tatsache nämlich, dass bei der Uranniere bei Durchspülung mit Normal-Ringer die NaCl-Konzentration im Ham in Vergleich mit derselben bei der gesunden Niere immer mehr zunimmt, kann im Sinne gedeutet werden, dass, wie oben erwähnt, die Steigerung der Filtration sowie die abnorm gesteigerte Rückresorption vorzüglich des Wassers aus den Tubulusepithelien zu starker Konzentrierung des Kochsalzes führen.

Bei der Kantharidinniere, deren Glomeruli bekanntermassen stark geschädigt sind, tritt die Steigerung der NaCl-Konzentration deshalb, weil hier zu erhöhter Kochsalzfiltration die funktionelle Störung der Tubulusepithelien hinzutritt und infolgedessen der Rückresorption des Wassers Vorschub geleistet wird, in weitgehend stärkerem Masse in Erscheinung, als bei der gesunden und Uranniere. Derartige Steigerungen der $\mathrm{NaCl}-$ Konzentration hören aber zu einer gewissen Zeit auf, was bei pathologischen Nieren überhaupt sehr verzögert eintritt.

In vorausgegangener Mitteilung ${ }^{11}$ ist bereits darüber ausführlich geschildert worden, durch was für einen Mechanismus die bei renoport-

15) Ya maguchi, Tohoku Journ. Exp. Med., 1926-1927, 8, 449; 1928, 9, 73, 274, 501 ฉ. $551 ; 1929,10,350$ u. $435 ; 1930,16,361$. 
aler Durchspülung mit kolloidaler Lösung vorkommende Rückresorption in den Tubulusepithelien ausgelöst wird; hier sei über den wesentlichen Grund dafür nochmals angeführt. Wenn man nämlich die Spülflüssigkeit, welche das Rinderserum, eine kolloidale Substanz, enthält, renoportal, also von dem Tubuluskapillarsystem aus hineinfliessen lässt, so stellt sich durch die Wirkung des erhöhten kolloid-osmotischen Drucks eine abnorme Steigerung der Rückresorption ein, welche selbstverständlicherweise bei bereits geschädigter Nierenepithelfunktion um so ausgeprägter in den Vordergrund tritt.

Bei renoportaler Durchspülung der gesunden Niere mit dem Rinderserum sollte die Filtration in den Glomeruli deshalb keine Veränderung erfahren, weil hierbei die glomeruläre Durchspülung mit NormalRinger ausgeführt wird; trotzdem kommt es schon von dem Anfang des zweiten Stadiums an zu erheblicher Oligurie und zur Erhöhung der Kochsalzkonzentration im Harn. Diese Koinzidenz der beiden Erscheinungen erklärt sich dadurch, dass die Nierenepithelien, wie oben bereits angeführt, durch den Einfluss der wasseraufsaugenden Kraft kolloidaler Lösung an ihrer Funktion, die Wasserrückresorption zu hemmen, eingeschränkt wurden, was zur auffallenden Oligohydrurie führte, die mit der Konzentrationsleistung für das Kochsalz einherging. Da hierbei die hemmende Kraft der Nierenepithelien auf die Kochsalzrückresorption vermutlich noch erhalten sein muss, erfährt der Kochsalzgehalt im Harn einen jähen Anstieg, und im übrigen ist hervorzuheben, dass die allmähliche Herabsetzung der Schwelle für $\mathrm{NaCl}$ in den Glomerulis begreiflicherweise als ein Hilfsmoment für die Koohsalzvermehrung mit im Spiel ist. Weil bei der Intaktheit der Niere aber, wenigstens im ersten Stadium der Durchspülung, die Schwelle im Glomerulus noch erhöht erhalten ist, kann der Uretertropfen das Kochsalz von dem Anbeginn an nicht konzentriert enthalten. Weil hier indessen die Harnmenge viel grösser ist, ist der NaCl-Gehalt dem absoluten Wert nach eher höher als bei der Uran- und Kantharidinniere.

Wenn man nun die prozentuelle Zunahme der Kochsalzkonzentration im ersten und zweiten Stadium bei der gesunden Niere gegeneinander vergleicht, so trifft man weitgehend höhere Werte als bei der Uran- und Kantharidinniere. In vorausgegangener Mitteilung ${ }^{1)}$ ist darauf aufmerksam gemacht worden, dass die geringfügige $\mathrm{Ab}$ nahme der Zufuhrmenge von der V. renoportalis aus, welche bei Durchspülung mit Rinderserum-Ringer im zweiten Stadium auftritt, durchaus nicht als ein Faktor für die Oligurie anzusprechen ist, und daraus kann man sich leicht vorstellen, dass diese Abnahme der Zufuhrmenge 
auch auf die $\mathrm{NaCl}-\mathrm{K}$ onzentration im Harn keinen Einfluss ausübt. Dass das Rinderserum vasokonstriktorisch wirkt, ist durch den Durchspïlungsversuch am Schenkelpräparat des Kaninchens von Sato ${ }^{16)}$ erwiesen worden. Diese Tatsache ist auch durch Durchströmungsversuche verschiedenster Art von Handovsky $u$. Pick ${ }^{17}$, Watanabeu. Odaira ${ }^{18)}$ Seto $^{19)}$ u.a. dargetan worden.

Bei der Uranniere, deren Funktion, die Rückresorption zu beschränken, sehr beeinträchtigt ist, tritt die Beeinflussung durch SerumRinger besonders eminent hervor, so dass der Rückresorptionsvorgang abnorm gesteigert ist. Aus diesem Grund ist es nicht zu vèrwundern, dass es schlisslich zu stärkster Oligurie kommt, welche am Ende des zweiten Stadiums ein beinahe an die Anurie angrenzendes Bild darbietet. Die NaCl-Konzentration erfährt im II. Stadium in analoger Weise wie bei der gesunden Niere bedeutende Steigerung, ist jedoch bald darauf, nach vorausgehender transitorischer Stabilität eher zum Absinken geneigt. Dies Phänomen dürfte höchst wahrscheinlich darauf zurückzuführen sein, dass bei pathologischen bzw. geschädigten Tubulusepithelien, weil hier die hemmende Kraft auf die Rückresorption zuvörderst des Wassers ausserordentlich abgeschwächt ist und danach die Hemmungswirkung a uf die Rückresorption anderer Harnbestandteile, hierbei also des $\mathrm{NaCl}$ immer intensiver beeinträchtigt ist, auch zusammen mit der Wasserrückresorption die Rückresorption des Kochsalzes gesteigert ist. Ein derartiges Phänomen setzt auch bei der Kantharidinniere andeutungsweise ein. Da in der Uranniere das Kochsalz schon von Anbeginn an reichlicher vorhanden ist, ist der Prozentsatz der NaCl-Konzentrationssteigerung weniger erheblich als bei der gesunden und Kantharidinniere. Auch die Oligurie tritt aufs intensivste in Erscheinung, weshalb die in der Zeiteinheit ausgeschiedene absolute $\mathrm{NaCl}-\mathrm{Menge}$ viel kleiner als bei der gesunden und Kantharidinniere ist.

Bei der Kantharidinniere, deren Glomeruli bekanntlich stärkste Schädigungen erleiden, ist die Filtration durch Funktionsherabsetzung derselben gesteigert, wobei auch der Vermehrung des Glomerulusharnes selbst entsprechend der $\mathrm{NaCl}-$ Gehalt zunimmt und zumal hierbei auch die Tubulusepithelien, die wenigstens funktionell gestört sind, im zweiten Stadium durch Serum-Ringer stark beeinflusst werden, er-

16) S a to, Tohoku Journ. Exp. Med., 1928, 9, 489.

17) Handovsky u. Pick, Arch. t. exp. Pathol. u. Pharm., 1913, 71, 61.

18) Watanabe u. Odaira, Tohoku Journ. Exp. Med., 1920, 1, 61.

19) Seto, Folia Pharm. Japon., 1928, 5, 259. 
höht sich die $\mathrm{NaCl}-\mathrm{Konzentration} \mathrm{des} \mathrm{Uretertropfens.} \mathrm{In} \mathrm{diesem} \mathrm{Fall}$ erweist sich die NaCl-Konzentration als solche am höchsten im Vergleich mit der gesunden und der Uranniere, wobei zugleich aber auch die in der Zeiteinheit ausgeschiedene absolute $\mathrm{NaCl}$-Menge am grössten ist. Die NaCl-Konzentration im Harn indessen ist deshalb, weil das Kochsalz seit dem Anfang des ersten Stadiums reichlicher als bei anderen Nierenarten präformiert vorhanden war, dem Prozentsatz nach berechnet, eher niedriger als bei der gesunden Niere. Nach alledem lässt sich sagen, dass bei pathologischen Nieren überhaupt die Reaktion auf die Spülfluissigkeit weniger ausgeprügt ist.

Bei renoportaler Durchspülung mit kolloidaler Lösung kommt es, gleichviel, ob es sich um gesunde oder um pathologische Nieren handle, stets zur Oligurie und zur Steigerung der $\mathrm{NaCl}-\mathrm{Konzentration.} \mathrm{Oben}$ angeführte Veränderungen treten um so augenscheinlicher hervor, je stärker die Nierenepithelschädigungen sind. Anderes gesprochen: durch den physiko-chemisch erhöhten osmotischen Druck des Spülflusssigkeit wird die Funktionsherabsetzung der Nierenepithelien verursacht, und namentlich bei pathologischen Nieren macht sich der Einfluss kolloidaler Substanzen, der Intensität der Schädigungen entsprechend, immer stärker geltend, so dass die Rückresorption physikochemisch in immer stärkerem Masse gefördert wird.

\section{Zusammenfassung.}

Es wurden isolierte überlebende Krötennieren in intakten sowie pathologischen Zuständen mit Rinderserum-Ringer von dem renoportalen System aus durchspült, während die Durchspülung von dem glomerulären System aus mit Normal-Ringer ausgeführt wurde; was zu folgenden Ergebnissen kam.

1. Es kommt stets unfehlbar zur Oligurie, gleichviel, ob es sich um gesunde oder um pathologische Nieren handelt. Diese Oligurie stellt sich bei der Uranniere im stärksten Grad ein, während sie bei der Kantharidinniere und der gesunden Niere der Reihe nach immer geringergradig zutage tritt.

2. Die Erhöhung der NaCl-Konzentration ist bei der gesunden Niere am auffallendsten, bei Kantharidinniere und Uranniere der Reihe nach immer unerheblicher.

3. Die in der Zeiteinheit ausgeschiedene absolute NaCl-Menge ist bei der gesunden Niere am grössten, bei der Kantharidinniere weniger gross und bei der Uranniere minimal.

Oben ausgeführte Versuchsergebnisse verschaffen eine Stütze für 
die Yamaguchis Ansicht, wonach die Funktionen der Nierenepithelien in regulierender bzw. hemmender Wirkung auf die Rückresorption bestehen.

Zum Schluss spreche ich Herrn Dr. Yamaguchi, dem seligen Dozenten an hiesiger Klinik, der mir bei meiner Arbeit mit Rat und Tat beigestanden hat, den herzlichsten Dank aus. 